\title{
The Dynamics of Phase Formation and Degradation of 2D Layered Hybrid Perovskites and Low-dimensional Hybrids Containing Mono-functionalized Oligothiophene Cations
}

\author{
Wouter T.M. Van Gompel, ${ }^{a}$ Paul-Henry Denis, ${ }^{a}$ Martijn Mertens, ${ }^{a}$ Bart Ruttens, ${ }^{c}$ Jan D'Haen, ${ }^{c}$ \\ ${ }^{\mathrm{a}}$ Kristof Van Hecke, ${ }^{\mathrm{b}}$ Laurence Lutsen, ${ }^{\mathrm{ac}}$ and Dirk Vanderzande*ac \\ aHybrid Materials Design (HyMaD), Institute for Materials Research (IMO-IMOMEC), Hasselt University, \\ Martelarenlaan 42, B-3500 Hasselt, Belgium

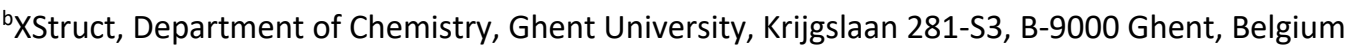 \\ 'Associated Laboratory IMOMEC, Imec, Wetenschapspark 1, B-3590 Diepenbeek, Belgium
}

Low-dimensional (2D or 1D) hybrid perovskites are receiving increased attention due to their structural flexibility and a generally enhanced stability compared to their 3D counterparts. Understanding the phase formation and degradation behavior of these materials is crucial towards their use in optoelectronic devices since different crystal phases possess different optical and electronic properties.[1-4]

In the first part of this talk, I will discuss the phase formation and degradation behavior of a series of lead iodide hybrids containing bithiophene, terthiophene and quaterthiophene derivatives. We show that two crystal phases can be formed for each of these systems, depending on the processing conditions. One of these phases corresponds to a $2 \mathrm{D}$ layered perovskite and the other phase has optical properties corresponding to a dimensionality intermediate between typical 2D and $1 \mathrm{D}$ hybrids. In the second part of this talk, I will discuss the influence of halide substitution on the phase formation of $2 \mathrm{D}$ layered perovskites (Bit-C3) ${ }_{2} \mathrm{PbX}_{4}$ (with $\mathrm{X}=\mathrm{Cl}, \mathrm{Br}$, and I), containing a bithiophene derivative (Bit-C3). The crystal structure and phase behavior of the 2D layered HOIPs were studied in detail. It is suggested that via halide substitution from iodide to bromide and chloride, the molecular degrees of freedom of the Bit-C3 ammonium cations are reduced by spatial confinement due to a smaller inorganic framework. Therefore, limiting the formation of lower-dimensional hybrids besides the targeted 2D layered HOIP.

1. Xiao, Z. W.; Meng, W. W.; Wang, J. B.; Mitzi, D. B.; Yan, Y. F., Searching for promising new perovskite-based photovoltaic absorbers: the importance of electronic dimensionality. Materials Horizons 2017, 4 (2), 206-216. 
2. Van Gompel, W. T. M.; Herckens, R.; Van Hecke, K.; Ruttens, B.; D'Haen, J.; Lutsen, L.; Vanderzande, D., Low-Dimensional Hybrid Perovskites Containing an Organic Cation with an Extended Conjugated System: Tuning the Excitonic Absorption Features. ChemNanoMat 2019, 5 (3), 323-327.

3. Van Gompel, W. T. M.; Herckens, R.; Denis, P.-H.; Mertens, M.; Gélvez-Rueda, M. C.; Van Hecke, K.; Ruttens, B.; D'Haen, J.; Grozema, F. C.; Lutsen, L.; Vanderzande, D., 2D layered perovskite containing functionalised benzothieno-benzothiophene molecules: formation, degradation, optical properties and photoconductivity. Journal of Materials Chemistry C 2020, 8 (21), $7181-7188$.

4. Van Gompel, W. T. M.; Herckens, R.; Van Hecke, K.; Ruttens, B.; D'Haen, J.; Lutsen, L.; Vanderzande, D., Towards 2D layered hybrid perovskites with enhanced functionality: introducing charge-transfer complexes via self-assembly. Chem Commun (Camb) 2019, 55 (17), 2481-2484. 